\title{
Robust PI, gain scheduled and PSS controller design using LMI regions: Comparative studies
}

\author{
Vojtech Vesely, Jan Murgas*
}

\begin{abstract}
This paper considers development of a new design procedure for PI and Gain scheduled robust controller with PSS for turbogenerator using LMI regional regions. The obtained PI+PSS and GSC+PSS robust controllers with output and state derivative feedback for uncertain polytopic turbogenerator model ensure that all closed -loop eigenvalues lay in the prescribed LMI region. The proposed method is to guarantee the less conservativeness with respect to previous methods due to introducing a design procedure based on new auxiliary matrices. The effectiveness of this procedure is illustrated by the examples of two controllers for turbogenerator.
\end{abstract}

K e y w ords: robust PI-PSS controller, gain scheduled controller, LMI region, state derivative feedback, output feedback, linear uncertain systems, $H_{2}$ performance

\section{Introduction}

Power system control is one of the most important control problems in electric power system (PS) design and operation, and is becoming more significant today due to of the increasing power and size, using new control techniques, changing structure of PS, environmental constraints, emerging new uncertainties, and the complexity of electric power system. The main goal of control of PS is to continuously supply electric power to all consumer with acceptable quality. In control of power system there are two main problems:

- to achieve feasible Voltage Profile using Automatic Voltage Regulator (AVR), and

- to achieve active power balance with feasible quality of frequency value, Automatic Load Frequency Control (ALFC).

To ensure the Global Asymptotic Stability (GAS) of power system using AVR and ALFC are of critical important for power stability/security. The controller design problem of automatic voltage regulator, ALFC and integrated control of AVR+ALFC in the last decade has been extensively studied and many controller design procedures are given in literature. The survey results could be summarized as follows.

Integrated approach to design of AVR and ALFC are given in $[1,9,10,15,29]$. For increase the GAS of power system the power system stabilizer (PSS) play very important role. In the PSS the output/state derivative feedback obviously has been used. The design procedure with different approaches has been find in [12,16$18,20,26,28]$. In these papers one can find to design of PSS using the quantitative Feedback Theory, LQR, frequency, Pareto method, linearizing method, Lyapunov function method, robust controller design and other approaches. Large number of papers and books devoted to AVR controller design. In [8] the nonlinear excitation control of a synchronous generator is proposed, [9] analytical method to design of excitation control, and in [10] two loop excitation control method is designed. Using observer approach the nonlinear AVR controller is designed for complex +multimachine power system in [27]. To disturbance attenuation in power system the nonlinear controller for turbogenerator has been designed in [11]. For turbogenerator controllers parameter tuning the simulation model of power system gives practically suitable results. For simulation of the power system plant the excellent books [21-23].

The above short observation of turbogenerator controllers design implies:

- because of nonlinear model to power system it demands use of corresponding controller design procedure;

- to guarantee GAS AVR and ALFC are of critical important for power system security;

- design procedures and calculation of controller parameters to AVR and ALFC on the main stream based on the linearized complex power system model;

- Lyapunov function approach or class of intelligent control is used to design of controller parameters when controller design procedure is based on nonlinear plant model of PS.

In this paper, using the idea of LMI region- regional pole placement method and/or $\mathrm{H}_{2}$ performance, gain scheduled controller and integrated controller design approach we have obtained the new controller design procedure for design of turbogenerator gain scheduled controllers. In the examples for comparative studies two

* Institute of Robotics and Cybernetics, Faculty of Electrical Engineering and Information Technology, Slovak University of Technology, Ilkovičova 3, Bratislava, Slovakia, vojtech.vesely@stuba.sk, jan.murgas@stuba.sk 
group of controllers will be designed: for the first group AVR the robust PI+PSS and ALFC governor control robust PI controller and for the second group AVR PI+PSS gain scheduled controller and ALFC governor control gain scheduled PI controller. Both group of controller has been design simultaneously for the nonlinear model of power system.

Gain scheduled control belongs to the most popular approached to control of Lipschitz nonlinear or linear parameter varying systems. LPV systems may considered as follows:

- they could be taken as LTI plants subject to gain scheduled time varying variables,

- they could be obtained as a set of LTI models resulting from linearization of the nonlinear model along the gain scheduled variables trajectory which we assume that are known.

The controller gain scheduled design procedure is based on Lyapunov function. The obtained theoretical, practical and simulation results show that the gain scheduled controller may give better quality to control of closed loop LPV system for all plant regimes than classical one including robust controller. The main results of gain scheduled controller design could be find in [24,31-35] and references therein.

Control objectives such as robust stabilization of uncertain systems, fast and well-damped of output variable, disturbance attenuation, satisfactory time responce can often be achieved by placing the closed loop poles in defined region of complex plain-regional pole placement. Regional pole placement often considered in conjunction with other performance criterion like as $H_{\infty}, L Q R$. Basic theory for using regional pole placement method in LTI systems are in $[1-3,7]$ and applications to power system in $[4,5]$.

Our notation is standard. Matrix $A \in R^{m \times n}$ marks a set of real matrices, $I_{m}$ is $m \times m$ identity matrix, $P>$ $0(P \geq 0)$ is a real symmetric, positive definite (semidefinite) matrix, $\otimes$ denotes Cronecker product, $1_{d} \in R^{d}$ is the vector with entries of one.

We present problem formulation and preliminaries, a short survey of LMI regions and basic definitions and lemmas originally presented in $[1,3]$. The main in the paper obtained results, gain scheduled controller design procedure with regional pole placement to LPV system.

We show that using minor modification of proposed method the regional pole placement approach and/or the $\mathrm{H}_{2}$ performance could be used. Numerical examples prove the effectiveness of the proposed approach, Section 5 provide comparative studies of two group designed turbogenerator controllers.

\section{Preliminaries and problem formulation}

Consider a linear continuous-time parameter varying uncertain system

$$
\dot{x}=A(\xi, \theta) x+B(\xi, \theta) u, y=C x
$$

where we assume that matrices with constant entries are affine with respect to scheduled vector parameter $\theta$ and uncertainty $\xi$, that is

$$
\{A(\xi, \theta), B(\xi, \theta)\}=\left(A_{0}(\xi), B_{0}(\xi)\right)+\sum_{i=1}^{p}\left(A_{i}(\xi), B_{i}(\xi)\right) \theta_{i}
$$

Assume that uncertain system belong to the polytope with $N$ vertices

$$
\begin{gathered}
\left\{A_{i}(\xi), B_{i}(\xi)\right\}=\sum_{j=1}^{N}\left(A_{i j}, B_{i j}\right) \xi_{j}, \sum_{j=1}^{N} \xi_{j}=1, \xi_{j} \geq 0 \\
\sum_{j=1}^{N} \dot{\xi}_{j}=0, \quad i=0,1,2, \ldots, p .
\end{gathered}
$$

Uncertainty $\xi_{j}, j=1,2, \ldots, N$ are constant or time varying unknown parameters, matrices $A_{i j}, B_{i j}, C$ are matrices of corresponding dimensions with constant entries, respectively, $\theta \in R^{p}$ is a known vector of constant or time varying gain scheduled parameters. We assume that both lower and upper bounds are available for gain scheduled parameters and their rates. Specifically,

- Each parameter $\theta_{i}, i=1,2, \ldots, p$ varies between known extremal values

$$
\theta_{i} \in \Omega_{p}=\left\{\theta_{i} \in\left\langle\underline{\theta}_{i}, \bar{\theta}_{i}\right\rangle, i=1,2, \ldots, p\right\} .
$$

- Rates of gain scheduled variable

$$
\dot{\theta}_{i} \in \Omega_{t}=\left\{\dot{\theta}_{i} \in\left\langle\dot{\theta}_{i}, \overline{\dot{\theta}}_{i}\right\rangle, i=1,2, \ldots, p\right\} .
$$

- Rates of uncertain parameter

$$
\dot{\xi}_{j} \in \Omega_{t \xi}=\left\{\dot{\xi}_{j} \in\left\langle\underline{\dot{\xi}}_{j}, \overline{\dot{\xi}}_{j}\right\rangle, j=1,2, \ldots, N\right\} .
$$

For PI controller design with static state/output feedback one need to append the plant state (1) with a new one to obtain plant output integral. Let us consider $\dot{z}=y=C x$. The following new state vector is obtained

$$
\begin{gathered}
\dot{x}_{n}=\left[\begin{array}{c}
\dot{x} \\
\dot{z}
\end{array}\right]=A_{n}(\xi, \theta) x_{n}+B_{n}(\xi, \theta) u, y_{n}=C_{n} x_{n} \\
A_{n}(\cdot)=\left[\begin{array}{cc}
A(\xi, \theta) & 0 \\
C & 0
\end{array}\right], \\
B_{n}(\cdot)=\left[\begin{array}{c}
B(\xi, \theta) \\
0
\end{array}\right], \quad C_{n}=\left[\begin{array}{cc}
C & 0 \\
0 & I
\end{array}\right] .
\end{gathered}
$$

The problem studied in this paper is to design both two robust PI controller for excitation and governor with power system stabilizer (PSS) and two robust gain scheduled PID controller with PSS for turbogenerator using regional pole placement approach [1] with $H_{2}$-performance by integral (QSR criterion, state, derivative of state and input) quadratic criterion. Control algorithm is in the form

$$
\begin{aligned}
& u=K(\theta) y_{n}+K_{d}(\theta) \dot{y}= \\
& {\left[\begin{array}{ll}
K_{p}(\theta) & \left.K_{i}(\theta)\right] y_{n}+\left[\begin{array}{ll}
K_{d}(\theta) & 0
\end{array}\right] \dot{y}_{n},
\end{array}\right.}
\end{aligned}
$$




$$
\left\{K(\theta), K_{d}(\theta)\right\}=X_{0}+\sum_{i=1}^{p} X_{i} \theta_{i} .
$$

Control algorithm (7) should ensure for closed loop system robustness, parameter dependent quadratic stability, minimal value of prescribed cost criterion and that all eigenvalues of closed-loop system are lying in the prescribed LMI region. Note we assume that the conditions to output feedback, see [6] are satisfied for uncertain gain scheduled plant model (6).

\section{Robust gain scheduled controller design}

\subsection{Short Review on LMI Regions}

Desired performance for control of closed-loop plant as minimal value of integral criterion, disturbance attenuation, time response, closed-loop damping, etc. can be reach by forcing the closed-loop eigenvalues into a defined region - regional LMI region in complex plane. In this subsection a necessary knowledged are given for LMI based characterization for a class of pole clustering LMI regional region with extended Lyapunov stability theorem for such LMI regions. For basic idea see [1-3]. An regional LMI region of the complex plane is any subset $D$-region that can be defined as $[3]$

$$
D=\left\{z \in C: L+z M+z^{\top} M^{\top}<0\right\}
$$

where $L$ is symmetric matrix and $M$ is real one. The characteristic functions of LMI $D$-region is as

$$
f_{D}(z)=L+z M+z^{\top} M^{\top}
$$

Some examples of LMI regions:

- left half complex plane

$$
\operatorname{Re}(z)<-\alpha, \quad \alpha \geq 0, \quad f_{D}(z)=z+z^{\top}+2 \alpha<0,
$$

- disk centered at $(-q, 0)$ and radius $r$

$$
f_{D}(z)=\left[\begin{array}{cc}
-r & q+z \\
q+z^{\top} & -r
\end{array}\right]<0
$$

- conic sector with apex at the origin of complex plane and inner angle $2 \eta$

$$
f_{D}(z)=\left[\begin{array}{ll}
\sin \eta\left(z+z^{\top}\right) & \cos \eta\left(z-z^{\top}\right) \\
\cos \eta\left(z^{\top}-z\right) & \sin \eta\left(z+z^{\top}\right)
\end{array}\right]<0
$$

Note that above conditions for LMI regions hold for linear time invariant (LTI) systems. When we assume that vector of uncertainty $\xi$ and gain scheduled variable $\theta$ are function of time the closed loop eigenvalues of LPV systems will lying in the prescribed LMI regions for all $\xi$ and $\theta$ only for the case of frozen above variables, that is for LTI systems. When the extended Lyapunov stability conditions for LPV systems are given then the time derivative of the Lyapunov function determines the prescribed LMI region boundary, that is all eigenvalues (for all $\xi, \theta)$ of closed loop system will lying in.

From uncertain model (6) and control algorithm (7) one obtain the closed-loop system in the form $\dot{x}_{n}=$ $A_{c}(\xi, \theta) x_{n}$. Assume that $A_{c}(\cdot)$ is convex with respect to uncertain vector parameter $\xi$ and vector gain scheduled variable $\theta$. Then from [1], Theorem 2.2, the following lemma is obtained

Lemma 1 The uncertain LTI system $A_{c}(\xi, \theta)$ is robustly $D$-region stable if positive definite matrix $P(\xi, \theta)$ exists such that

$$
L \otimes P(\cdot)+M \otimes\left(P(\cdot) A_{c}(\cdot)\right)+M^{\top} \otimes\left(A_{c}(\cdot)^{\top} P(\cdot)\right)<0
$$

for $\forall(\theta) \in \Omega_{p}, \forall(\xi) \in \Omega_{\xi}, P(\xi, \theta)>0$.

Multiplying from left and right hand side of (10) by $x_{n}^{\top}, x_{n}$, using closed-loop plant model and taking into account the extended Lyapunov stability of LPV systems one obtains

$$
\begin{aligned}
L \otimes\left(x_{n}^{\top}(P(.)+\dot{P}(\cdot)) x_{n}\right)+M & \otimes\left(x_{n}^{\top} P(\cdot) \dot{x}_{n}\right)+ \\
& M^{\top} \otimes\left(\dot{x}_{n}^{\top} P x_{n}\right)<0
\end{aligned}
$$

which can be rewrite in matrix form as follows

$$
\begin{gathered}
{\left[\left(1_{d} \otimes x_{n}\right)^{\top}\left(1_{d} \otimes \dot{x}_{n}\right)^{\top}\right]\left[\begin{array}{cc}
L \otimes P(\cdot)+L \otimes \dot{P}(\cdot) * \\
M^{\top} \otimes P(\cdot) & 0
\end{array}\right] v_{1} \leq 0} \\
v_{1}^{\top}=\left[\begin{array}{cc}
\left(1_{d} \otimes x_{n}\right)^{\top} & \left.\left(1_{d} \otimes \dot{x}_{n}\right)^{\top}\right] .
\end{array}\right.
\end{gathered}
$$

Symbol $\otimes$ is the Kronecker product, $I_{d}$ is unit matrix with dimension $d, 1_{d} \in R^{d}$ is the vector with entries of one, $M \in R^{d \times d}, \dot{P}(\cdot)$ the first derivative of Lyapunov matrix $P(\xi, \theta)$, (see (16)). From (11) and (13) one obtains the first new results of the paper.

Lemma 2 The uncertain LPV system $A_{c}(\xi, \theta)$ is robustly D-region stable for all $(\xi, \theta, \dot{\theta}, \dot{\xi})$ if the inequality (12) holds for uncertain closed-loop system and $\forall(\theta) \in \Omega_{p}, \forall(\dot{\theta}) \in \Omega_{t}, \forall(\xi) \in \Omega_{\xi}, \forall(\dot{\xi}) \in \Omega_{t \xi}, P(\xi, \theta)>0$.

Note that using inequalities (12), (6) and (7) one can obtain the time derivative of extended Lyapunov function to LMI $D$-region and LPV systems for general characteristic equation given by (9).

\subsection{Robust Gain Scheduled Controller Design for turbo- generator}

The notion of parameter dependent Ljapunov functionquadratic stability (PDQS) is useful tool for analyzing and robust gain scheduled controller designing to uncertain state space models. In this subsection using extended parameter dependent Lyapunov function to design of robust gain scheduled controller which ensure PDQS for arbitrary LMI regions given by matrices $L$ and $M$ is derived. After small modification of obtained results one can introduce $\mathrm{H}_{2}-Q S R$ performance to robust gain scheduled controller design. Let us consider the Lyapunov function

$$
V(\xi, \theta)=x_{n}^{\top} P(\xi, \theta) x_{n}
$$


where for the gain scheduled Lyapunov matrix holds

$$
\begin{gathered}
P(\xi, \theta)=P_{0}(\xi)+\sum_{i=1}^{p} P_{i}(\xi) \theta_{i}, \quad P_{i}(\xi)=\sum_{j=1}^{N} P_{i j} \xi_{j}, \\
i=0,1,2, \ldots, p .
\end{gathered}
$$

Time derivative of (13) for LPV model gives

$$
\begin{gathered}
\frac{\mathrm{d} V(\cdot)}{\mathrm{d} t}=\dot{x}_{n}^{\top} P(\xi, \theta) x_{n}+x_{n}^{\top} \dot{P}(\xi, \theta) x_{n}+x_{n}^{\top} P(\xi, \theta) \dot{x}_{n} \\
=v^{\top}\left[\begin{array}{ccc}
\dot{P}(\xi, \theta) & P(\xi, \theta) & 0 \\
P(\xi, \theta) & 0 & 0 \\
0 & 0 & 0
\end{array}\right] v \\
\dot{P}(\xi, \theta)=\sum_{j=1}^{N}\left[\sum_{k=1}^{N} P_{o k} \dot{\xi}_{k}+\sum_{i=1}^{p} \sum_{k=1}^{N} P_{i k} \dot{\xi}_{k} \theta_{i}+\sum_{i=1}^{p} P_{i j} \dot{\theta}_{i}\right] \xi_{j} \\
=\sum_{j=1}^{N}\left(d P_{2}+d P_{1}\right) \xi_{j}+\sum_{j=1}^{N}\left(\sum_{i=1}^{p} d P_{3} \theta_{i}\right) \xi_{j}, \\
v^{\top}=\left[\begin{array}{ll}
x_{n}^{\top} & \dot{x}_{n}^{\top} \\
u^{\top}
\end{array}\right] .
\end{gathered}
$$

In (14) the equality $\sum_{j=1}^{N} \xi_{j}=1$ has been used and

$$
\begin{gathered}
\mathrm{d} P_{1}=\sum_{i=1}^{p} P_{i j} \dot{\theta}_{i}, \quad \mathrm{~d} P_{2}=\sum_{k=1}^{N} P_{o k} \dot{\xi}_{k} \\
\mathrm{~d} P_{3}=\sum_{i=1}^{p} \sum_{k=1}^{N} P_{i k} \dot{\xi}_{k} .
\end{gathered}
$$

To guarantee that closed loop system matrix $A_{c}(\xi, \theta)$ is convex with respect to uncertainty $\xi$ and gain scheduled variable $\theta$, split plant systems and Lyapunov matrices and to reduce the conservativeness of controller design procedure we have introduced to the first derivative of extended Lyapunov function auxiliary matrices $N_{1}, \ldots, N_{6}$ as follows

$$
\begin{aligned}
& v^{\top}\left[\begin{array}{l}
2 N_{1}^{\top} \\
2 N_{2}^{\top} \\
2 N_{3}^{\top}
\end{array}\right]\left[-A_{n}(\xi, \theta) \quad I \quad-B_{n}(\xi, \theta)\right] v=0,
\end{aligned}
$$

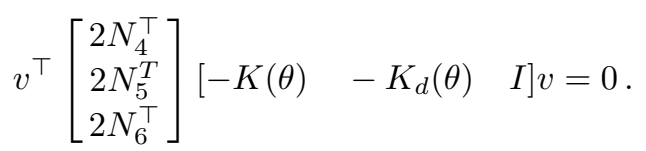

Summarizing equations (15) and (12) for the time derivative of extended Lyapunov function we have

$$
\begin{gathered}
\frac{d V_{\text {ext }}}{d t}=\sum_{j=1}^{N} v_{d}^{T} W_{j}(\theta) v_{d} \xi_{j}<0 \rightarrow \\
W_{j}(\theta)=W_{0 j}+\sum_{i=1}^{p} W_{i j} \theta_{i}<0 \quad W_{0 j}=\left\{w_{0 k l}\right\}_{3 \times 3} \\
W_{i j}=\left\{w_{i k l}\right\}_{3 \times 3}, j=1,2, \ldots, N, i=1,2, \ldots, p \\
v_{d}^{\top}=\left[\left(1_{d} \otimes x_{n}\right)^{\top} \quad\left(1_{d} \otimes \dot{x}_{n}\right)^{\top} \quad\left(1_{d} \otimes u\right)^{\top}\right]
\end{gathered}
$$

where

$$
\begin{aligned}
& w_{011}=L \otimes\left(P_{0 j}+d P_{2}+d P_{1}\right)-N_{1}^{\top}\left(I_{d} \otimes A_{n 0 j}-\right. \\
& \left(I_{d} \otimes A_{n o j}^{\top} N_{1}-N_{4}^{\top}\left(I_{d} \otimes K_{0} C_{n}\right)-\left(I_{d} \otimes K_{0} C_{n}\right)^{\top} N_{4}\right. \text {, } \\
& w_{012}=M^{\top} \otimes P_{0 j}+N_{1}^{\top}-\left(I_{d} \otimes A_{n 0 j} N_{2}-\right. \\
& N_{4}^{\top}\left(I_{d} \otimes K_{d 0}-\left(I_{d} \otimes K_{0} C_{n}\right)^{\top} N_{5},\right. \\
& w_{013}=-\left(I_{d} \otimes A_{n 0 j}^{\top} N_{3}-N_{1}^{\top}\left(I_{d} \otimes B_{n 0 j}+\right.\right. \\
& N_{4}^{\top}-\left(I_{d} \otimes K_{0} C_{n}\right)^{\top} N_{6}, \\
& w_{023}=-N 2^{\top}\left(I_{d} \otimes B_{n 0 j}+N_{3}+N_{5}^{\top}-\left(I_{d} \otimes K_{d 0}^{\top} N_{6},\right.\right. \\
& w_{022}=N_{2}^{\top}+N_{2}-N_{5}^{\top}\left(I_{d} \otimes K_{d 0}-\left(I_{d} \otimes K_{d 0}^{\top} N_{5},\right.\right. \\
& w_{033}=-N_{3}^{\top}\left(I_{d} \otimes B_{n 0 j}-\left(I_{d} \otimes B_{n 0 j}^{\top} N_{3}+N_{6}+N_{6}^{\top},\right.\right. \\
& w_{i 11}=L \otimes\left(P_{i j}+\mathrm{d} P_{3}\right)-N_{1}^{\top}\left(I_{d} \otimes A_{n i j}-\left(I_{d} \otimes A_{n i j}^{\top} N_{1}\right.\right. \\
& -N_{4}^{\top}\left(I_{d} \otimes K_{i} C_{n}\right)-\left(I_{d} \otimes K_{i} C_{n}\right)^{\top} N_{4}, \\
& w_{i 12}=M \otimes P_{i j}-\left(I_{d} \otimes A_{n i j}^{\top} N_{2}-N_{4}^{\top}\left(I_{d} \otimes K_{d i}-\right.\right. \\
& \left(I_{d} \otimes K_{i} C_{n}\right)^{\top} N_{5} \\
& w_{i 13}=-\left(I_{d} \otimes A_{n i j}^{\top} N_{3}-N_{1}^{\top}\left(I_{d} \otimes B_{n i j}-\right.\right. \\
& \left(I_{d} \otimes\left(K_{i} C_{n}\right)\right)^{\top} N_{6}, \\
& w_{i 23}=-N_{2}^{\top}\left(I_{d} \otimes B_{n i j}-\left(I_{d} \otimes K_{d i}^{\top} N_{6},\right.\right. \\
& w_{i 22}=-N_{5}^{\top}\left(I_{d} \otimes K_{d i}-\left(I_{d} \otimes K_{d i}^{\top} N_{5},\right.\right. \\
& w_{i 33}=-N_{3}^{\top}\left(I_{d} \otimes B_{n i j}-\left(I_{d} \otimes B_{n i j}\right) T N_{3},\right. \\
& i=1,2, \ldots, p, j=1,2, \ldots, N \text {. }
\end{aligned}
$$

Without changing the denotation for new extended auxiliary variable $N_{i}, i=1,2, \ldots, 6$ in (16) holds $N_{i}=$ $N_{i \text { new }}=I_{d} \otimes N_{i \text { old }}$. The obtained main results of this paper (design procedure for design of robust gain scheduled controller) are summarized in the next theorem.

TheOREM 1. An uncertain LPV system (6) with the control gain scheduled algorithm (7) is parameter dependent quadratic $D$-region stable with eigenvalues of closed-loop system lying in the prescribed LMI D-region if there positive definite matrix $P(\xi, \theta)$ (14), auxiliary matrices $N_{1}, N_{2}, \ldots, N_{6}$, gain scheduled controller matrices $K(\theta), K_{d}(\theta)$ exist such that inequality (18) holds for all $j=1,2, \ldots, N, i=0,1,2, \ldots, p, \theta \in \Omega_{p}, \dot{\theta} \in \Omega_{t}$, $\dot{\xi} \in \Omega_{t \xi}, \forall(\xi) \in \Omega_{\xi}$.

Proof . For concrete structure of Lyapunov function (14) one obtains the sufficient parameter dependent quadratic $D$-region stability condition, therefore proof of the above theorem immediately follows from (12), (13), (14) and (15).

R e m a r k 1 . Due to auxiliary matrices the inequality (16) is convex with respect to $(\xi, \theta)$, therefore for guarantee the parameter dependent quadratic $D$-region stability of closed loop system it is sufficient if (18) holds for all $\xi, \dot{\xi}, \theta, \dot{\theta}$ vertices. 
$\mathrm{R}$ e $\mathrm{m}$ a r k 2 . Note that if in entries of $w_{011}$ and $w_{i 11}$ for all $i=1,2, \ldots, p$, holds $P_{i}(\xi)>0$, then instead of $\dot{\xi}, \dot{\theta}$ one can set their maximal values.

$\mathrm{R}$ e $\mathrm{m}$ a $\mathrm{rk} 3$. In the case when one with regional pole placement method simultaneously want to use the $H_{2}$ performance with cost function

$$
J_{c}=\int_{0}^{\infty}\left(x_{n}^{\top} Q x_{n}+\dot{x}_{n}^{\top} S \dot{x}_{n}+u^{\top} R u\right) \mathrm{d} t
$$

where $Q, S$ are positive definite (semidefinite) and $R$ is positive definite matrices, respectively. In this case it need to change the following entries of matrix $W_{0 j}$ as follows $w_{011}^{n}=w_{011}+I_{d} \otimes Q, w_{022}^{n}=w_{022}+I_{d} \otimes S, w_{033}^{n}=$ $w_{033}+I_{d} \otimes R$. If one wants to use more sophisticated $Q S R$ criterion as $(Q, S, R)=X_{0}+\sum_{i=1}^{p} X_{i} \theta_{i}$ it is easy to follow where one need to set the matrices $X_{0}, X_{i}$ to the corresponding part of $W_{0 j}, W_{i j}$.

$\mathrm{R}$ e $\mathrm{mark} 4$. For the case only of robust controller design with LMI $D$-region pole placement approach (and $H_{2}$ performance) it is sufficient to set $p=0$.

$\mathrm{Remark} 5$. For the case of switched robust gain scheduled controller design with arbitrarily switching algorithm with regional LMI $D$-region pole placement approach and/or $H_{2}$ performance it is enough to set $\overline{\dot{\theta}}_{i}$, $i=1,2, \ldots, p$ for ideal switching $\infty$ and for non ideal switching sufficient large value corresponding to the rate of switching variable.

\section{Example}

In this example, at the first one transform the turbogenerator nonlinear model to the linear parameter varying system (LPV). The nonlinear model of turbogenerator [21-23], which consists of nonlinear 3rd order model of synchronous generator (SG) and linear thermal turbine model, under the well-known assumptions are described as follows

- 3rd order synchronous generator nonlinear model:

$$
\begin{gathered}
\left(E_{q}, U_{d}, U_{q}, U_{b}, P_{e}\right) \quad \text { in } \mathrm{pu} \\
U_{q}=I_{d} X_{d}+E_{q}-I_{q} R_{a}, \\
U_{d}=-I_{q} X_{q}-I_{d} R_{a}, \\
U_{b} k_{G 0}=E_{q}+T_{d 0}^{\prime} \frac{\mathrm{d} E_{q}}{\mathrm{~d} t}+T_{d 0}^{\prime} \frac{\mathrm{d} I_{d}}{\mathrm{~d} t}\left(X_{d}-X_{d}^{\prime}\right), \\
T_{j} \frac{\mathrm{d} \omega}{\mathrm{d} t}=P_{T}-P_{e}, \\
P=P_{e}=P+P_{a s}, \quad P_{a s} \doteq D \omega, \\
P=E_{q} I_{q}+I_{d} I_{q}\left(X_{d}-X_{q}\right)-R_{a}\left(I_{d}^{2}+I_{q}^{2}\right) .
\end{gathered}
$$

where $I_{d}, I_{q}$ - currents running stream in the fictitious $d$ and $q$ axis armature coils,
$E_{q}-q$-axis voltage of the internal emf, proportional to the excitation current of SG,

$U_{q}, U_{d}$ - voltages across the fictitious $d$ and $q$ axis armature coils,

$P_{e}, P_{a s}$ - total SG active power generated to the power system, and damping power,

$U_{b}$ - SG excitation voltage applied to the field winding, input variable of $\mathrm{SG}$

$T_{d 0}^{\prime}-d$-axis transient time constant for the case of opencircuit,

$T_{j}$ - turbogenerator inertia coefficient,

$X_{d}, X_{d}^{\prime}, X_{q}$ - reactance and transient reactance of the fictitious $d$ and $q$ axis winding,

$R_{a}$ - armature winding resistance,

$\left.U=\sqrt{(} U_{d}^{2}+U_{q}^{2}\right)-$ SG terminal voltage.

The load angle of rotor SG time derivative $\delta$

$$
\frac{\mathrm{d} \delta}{\mathrm{d} t}=\Delta \omega=\omega-\omega_{s}
$$

is the SG rotor speed deviation in $\mathrm{rad} / \mathrm{s}$ and $\omega_{s}-$ power system angular speed. In the following we assume that $\omega_{s}=$ const. therefore we will use the denotation $\Delta \omega=\omega$. For the case of simplified structure thermal turbine linear model with governor valve [19] is given by the third order transfer function

$$
G_{T(s)}=\frac{P_{T(s)}}{P_{R(s)}}=\frac{s b_{1}+b_{0}}{s^{3} a_{3}+s^{2} a_{2}+s a_{1}+1} .
$$

where $P_{T(s)}$ - turbine power output,

$P_{R(s)}$ - turbine power controller output,

$$
\begin{aligned}
& b_{1}=k_{l} T_{h}+k_{h} T_{l}, \quad b_{0}=k_{l}+k_{h}=1, \quad a_{3}=T_{s} T_{l} T_{h}, \\
& a_{2}=T_{l} T_{h}+T_{s}\left(T_{l}+T_{h}\right), \quad a_{1}=T_{l}+T_{h}+T_{s} .
\end{aligned}
$$

Basic values of the thermal turbine parameters are: servomotor time constant $T_{s}=0.1 \mathrm{~s}$, low and high pressure gains and time constants $k_{l}=0.7 \mathrm{p} . \mathrm{u}, T_{l}=4-11 \mathrm{~s}, k_{h}=0.3 \mathrm{p} . \mathrm{u}, T_{h}=0.1 \mathrm{~s}$.

In general, power system turbogenerators are always subject to disturbances. Bring up against the disturbances and calculate the stability of complex power systems in [30] the One-Machine-to-Quasi-Infinite Bus System has been introduced. In the following we take the idea of [30] we will study a single-one machine power system connected to a complex power system through transmission lines to a bus $\{$ voltage, angular speed $\}=$ $\left(U_{s}, \omega_{s}\right)[22,23]$. We suppose that because of the relative large size of the power system to our machine which supplying power, the dynamics of our system no changes the voltage $U_{s}$ and frequency $\omega_{s}$. Transmission lines one should transformed to the $T$ equivalent circuit with impedance $\bar{z}_{1}, \bar{z}_{2}$, and reluctant impedance $\bar{z}_{3}$. On the base of Kirchhoff's laws one can obtains for currents on the $d$ and $q$ axis of SG as follows.

$$
I_{d}=-\frac{E_{q}}{M}+\frac{U_{s}}{z_{12}\left(1+\frac{X_{d}-X_{q}}{z_{11}} \sin \varphi_{11}\right.} \sin \left(\delta+\varphi_{12}\right)
$$




$$
\begin{aligned}
I_{q}=\frac{E_{q}}{M \tan \varphi_{11}}+ & \frac{U_{s}}{z_{12}} \sqrt{1+\left[\frac{\left(X_{d}-X_{q}\right) \cos \varphi_{11}}{z_{11}+\left(X_{d}-X_{q}\right) \sin \varphi_{11}}\right]^{2}} \\
& \times \sin \left(\delta+\varphi_{12}-\psi\right)
\end{aligned}
$$

where

$$
\begin{gathered}
M=\frac{z_{11}}{\sin \varphi_{11}}+X_{d}-X_{q}, \\
\psi=\arctan \frac{z_{11}+\left(X_{d}-X_{q}\right) \sin \varphi_{11}}{\left(X_{d}-X_{q}\right) \cos \varphi_{11}}, \\
\bar{z}_{11}=j X_{q}+\bar{z}_{1}+\frac{\bar{z}_{2} \bar{z}_{3}}{\bar{z}_{2}+\bar{z}_{3}}=z_{11} e^{j \varphi_{11}}, \\
\bar{z}_{12}=j X_{q}+\bar{z}_{1}+\frac{\bar{z}_{2}}{\bar{z}_{3}}\left(\bar{z}_{3}+\bar{z}_{1}+j X_{q}\right)=z_{12} e^{j \varphi_{12}}
\end{gathered}
$$

\subsection{Design of LPV model to turbogenerator}

Now, we are about ready to obtain linear parameter varying system (LPV) from (18)-(20). For details one should consults at the survey paper [31]. LPV system model or gain scheduled plant model of turbogenerator can be obtained by following steps:

1. Choose the measurable vector of gain scheduled variable, which allow for parametric dependence of the turbogenerator (nonlinear) plant model, for this case we will choose gain scheduled variable as active power $P(\mathrm{pu})$.

2. Designate the values of gain scheduled variable (working point) where the LPV model and nonlinear model have to be equal, in our case $(w=1,2,3, P=$ $(0.3,0.7,1)(\mathrm{pu}))$. For chosen three working points one need $p=2$ gain scheduled variables

3. At each chosen working points, one builds the linearized model of turbogenerator

$$
\dot{x}=\overline{A_{w}} x+\overline{B_{w}} u \quad w=1,2,3, \quad y=C x
$$

where $x(t) \in R^{n}$ - state vector of turbogenerator, $u(t) \in R^{m}$ - control, input vector, $y(t) \in R^{l}$ - output to be controlled vector of system

4. Introduce gain scheduled model of turbogenerator. Let the gain scheduled time varying parameters $\theta_{j}, j=1,2$ be a function of active power $\theta_{j}=f(P), j=1,2$.

$$
\begin{gathered}
\dot{x}=A(\theta) x+B(\theta) u, \quad y=C x, \\
A(\theta)=A_{0}+\sum_{j=1}^{p} A_{j} \theta_{j} \in R^{n \times n}, \\
C \in R^{l \times n} \\
u=\left[\begin{array}{ll}
\Delta U_{b} & \Delta P_{R}
\end{array}\right]^{\top}, \quad y=\left[\begin{array}{ll}
\Delta U & \Delta P
\end{array}\right]^{\top} .
\end{gathered}
$$

In (24) $\theta \in R^{p}$ - vector of known constant or time varying gain scheduled parameters.

5. For linearized points $w=1,2,3$ one should extremal values of gain scheduled parameters substitute to (24). Assume that extremal values of above parameters are $\theta_{j}=-1$ or $\theta_{j}=1$ for $j=1,2$. Note that in our case $\theta_{1}=-1$ and $\theta_{2}=1$ does not exist. For working points $w=1,2,3$ compare the obtained results with (23) one have got

$$
\begin{aligned}
& \bar{A}_{1}=A_{0}-A_{1}-A_{2}, \theta_{1}=-1, \theta_{2}=-1, w=1, \\
& \bar{A}_{2}=A_{0}+A_{1}-A_{2}, \theta_{1}=1, \theta_{2}=-1, w=2, \\
& \bar{A}_{3}=A_{0}+A_{1}+A_{2}, \theta_{1}=1, \theta_{2}=1, w=3 .
\end{aligned}
$$

From (25) one obtains the simple matrix equation for calculation of gain scheduled model (24) in the form

$$
\left[\begin{array}{rrr}
I & -I & -I \\
I & I & -I \\
I & I & I
\end{array}\right]\left[\begin{array}{l}
A_{0} \\
A_{1} \\
A_{2}
\end{array}\right]=\left[\begin{array}{l}
\bar{A}_{1} \\
\bar{A}_{2} \\
\bar{A}_{3}
\end{array}\right]
$$

For ensured I-part gain scheduled controller design the states of the original system (24) need to be append, for more detail see [25]. The control algorithm for PID controller can be written as follows

$$
u(t)=K_{p} y(t)+K_{i} \int_{0}^{t} C x(\tau) \mathrm{d} \tau+K_{d} \dot{y}(t .)
$$

Integral term should be included into the state vector defining the new auxiliary state as $z=\int_{0}^{t} x(\tau) \mathrm{d} \tau$ for this case PID control algorithm is

$$
\begin{aligned}
& u(t)=K_{p} C x(t)+K_{i} C z(t)+K_{d} C_{d} \dot{x}(t)= \\
& {\left[\begin{array}{ll}
K_{p} & K_{i}
\end{array}\right] C\left[\begin{array}{c}
x(t) \\
z(t)
\end{array}\right]+\left[\begin{array}{ll}
K_{d} C_{d} & 0
\end{array}\right]\left[\begin{array}{c}
\dot{x}(t) \\
\dot{z}(t)
\end{array}\right] .}
\end{aligned}
$$

With new auxiliary plant state the new plant state and output vectors are given as $X(t)=\left[x(t)^{\top}, z(t)^{\top}\right]^{\top}$, $Y(t)=\left[y(t)^{\top}, C z(t)^{\top}\right]^{\top}$, when the controller has no integral term the plant state and output does not change. In the following, for all type of controller structure, we will assume that denotation of plant state and output vectors $x(t)=X(t), y(t)=Y(t)$, matrices and matrices dimensions do not changes and the static output feedback control algorithm can provide the proportional and integral parts of the designed robust gain scheduled PID controller using (24).

The the following problem is studied. Design both a robust output feedback $P I+P S S$ robust controller for excitation and governor control and gain scheduled $P I+P S S$ controller such that all eigenvalues of closed loop system will lying in the prescribed LMI region with control algorithm

$$
u=K(\theta) y+K_{d}(\theta) \dot{y}=K(\theta) C x+K_{d}(\theta) C_{d} \dot{x}
$$

where for gain scheduled controller $K(\theta)=K_{0}+$ $\sum_{j=1}^{p} K_{j} \theta_{j}=\left[\begin{array}{ll}K_{p}(\theta) & K_{i}(\theta)\end{array}\right] \in R^{m \times 2 l} \quad K_{d}(\theta)=K_{d 0}+$ $\sum_{j=1}^{p} K_{d j} \theta_{j} \in R^{m \times l}, C_{d} \in R^{l \times n}$ is the SG output matrix for the PSS (derivative) part of controller, such that the controller guarantee that all closed-loop eigenvalues 


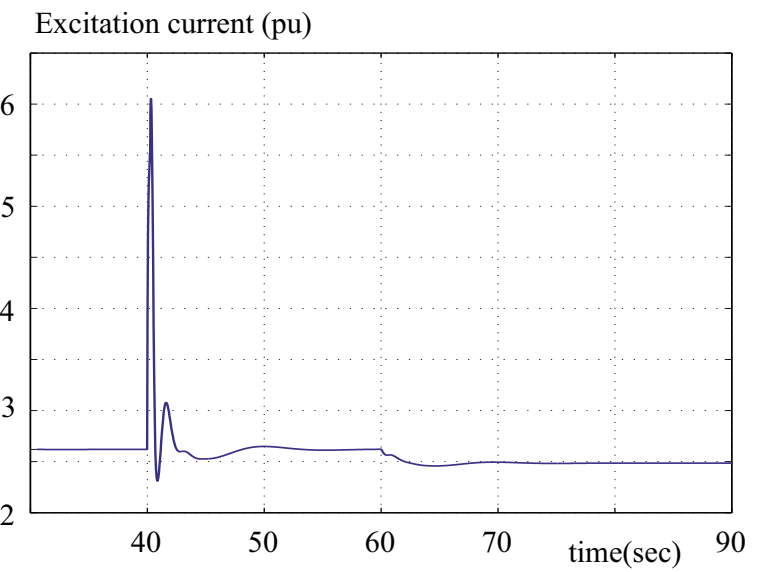

Fig. 1. Excitation current under disturbances-PI

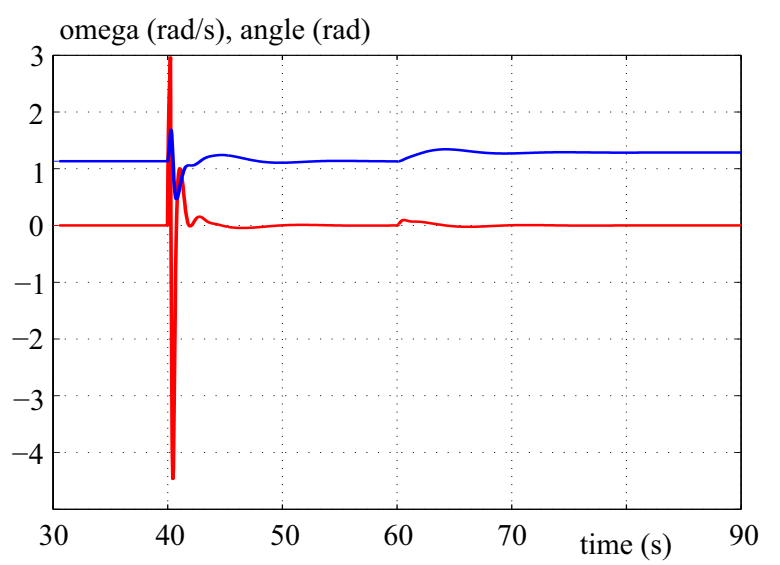

Fig. 3. Frequency changes and load angle under disturbances-PI

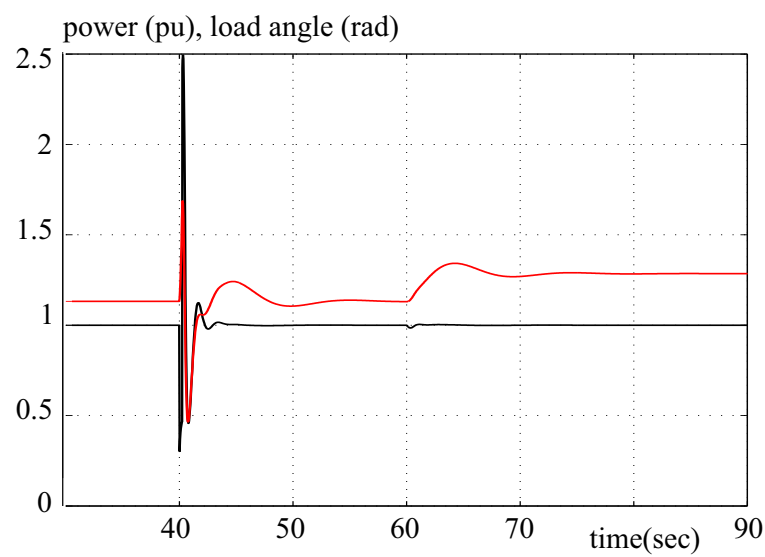

Fig. 5. Terminal voltage of SG dynamics under disturbances-GSC

lying in the prescribed LMI region, robust PDQS and guaranteed cost with respect to the closed loop system, $((24)+(27))$. As a PSS controller excitation of SG we will used the first derivative of load angle.

Parameters of turbogenerators and transmission lines are as follows:

$$
\begin{aligned}
& T_{j}=0.02245 \mathrm{~s}^{2}, T_{d 0}^{\prime}=0.4 \mathrm{~s}, \\
& X_{d}=2 \mathrm{pu}, X_{d}^{\prime}=0.247 \mathrm{pu} \\
& X_{q}=1.75 \mathrm{pu}, R_{a}=0, \\
& X_{v 1}=0.127 \mathrm{pu}, X_{v 2}=0.12 \mathrm{pu}
\end{aligned}
$$

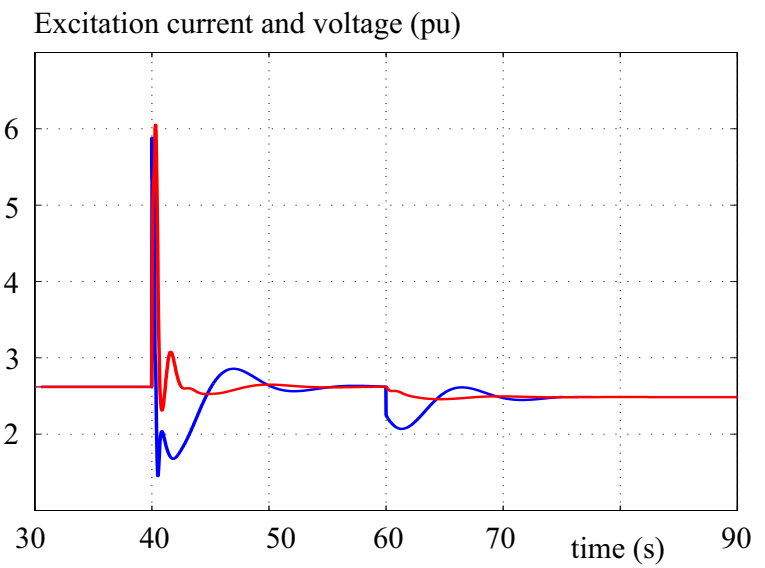

Fig. 2. Excitation current and controller output voltage under disturbances-PI

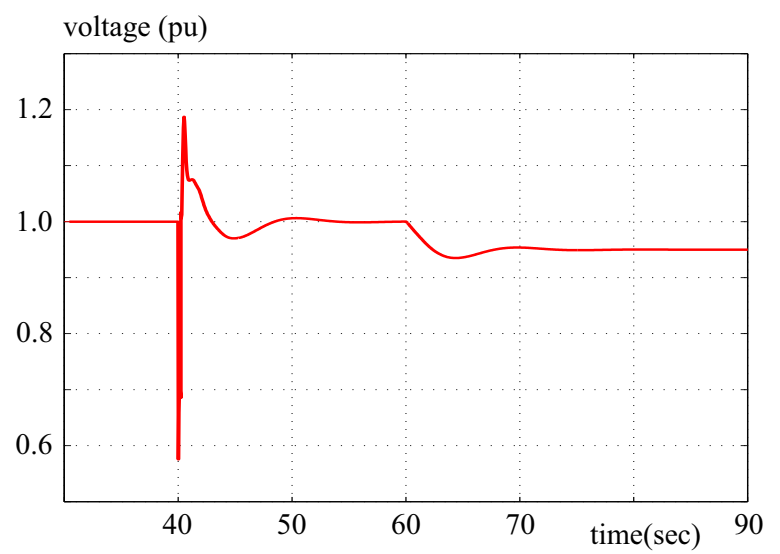

Fig. 4. Load angle dynamics under described disturbances-GSC

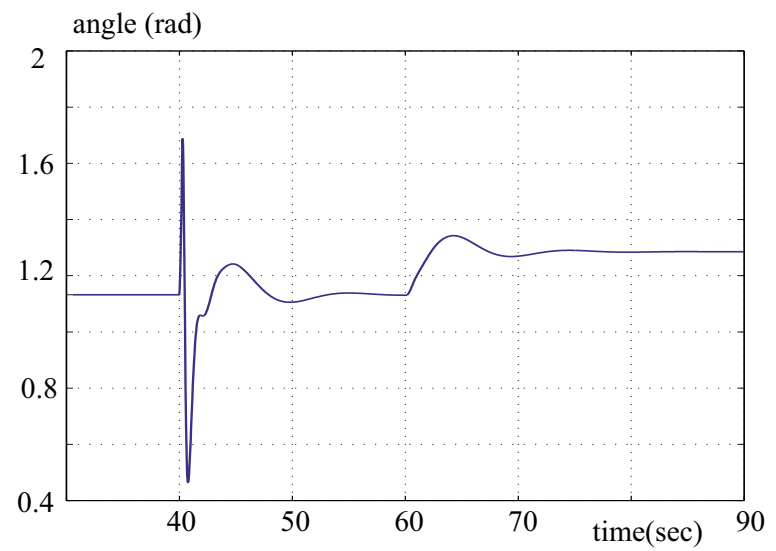

Fig. 6. Gain scheduled variables under dynamic disturbances-GSC

Thermal turbine:

$$
\begin{aligned}
& T_{s}=0.4 s, T_{l}=5.4 s, \\
& T_{h}=0.25, k_{l}=0.75, k_{n}=0.25
\end{aligned}
$$

Working points are given as $\left(U_{z}, P_{z}-\right.$ which serve as set points of terminal voltage and electrical active power).

$$
U_{z}=1 \mathrm{pu}, P_{z}=1 \mathrm{pu}, P=\langle 0.3,0.7,1\rangle \mathrm{pu}
$$

Gain scheduled variables and other parameters

$$
\theta_{i} \in\langle-1,1\rangle,\|P(\theta)\| \leq \text { ro }=100000
$$


The rate of gain scheduled parameter changes and plant parameter changes are given $\dot{\theta}=0.2 / \mathrm{sec}$ and $\dot{\xi}=0.00005 / \mathrm{sec}$. Note that the matrix dimensions of turbogenerator state, input and output matrices are $A \in$ $R^{8 \times 8}, B \in R^{8 \times 2}, C \in R^{4 \times 8}, C_{d} \in R^{4 \times 8}$ and output $y=\left[\begin{array}{ll}y_{p} & y_{i}\end{array}\right]$ where $y_{p}, y_{i}$ are output of terminal voltage and active power for proportional controller and respectively integral of terminal voltage and active power for integral part of controller. Derivative part controller output is $\dot{y}_{d}=\dot{\delta}$ which represents the derivative of load angle of SG. Demanded performance of closed loop system are given by two criterion at the first all eigenvalues of closed loop system need to be lying on the LMI region given as the moved complex axis to degree of stability $\alpha=0.6$ of complex plane and $H_{2}$ integral criterion known as QRS (state, input and derivative of state), where $Q=0.00001 * I_{n}, S=0.00001 * I_{n}, R=I_{m}$.

The subsequent two simulation experiments are have been made for designed two controllers:

- at time $\mathrm{t}=40 \mathrm{~s}$ two phase short circuits were realized in the power system such that within the time $=0.2 \mathrm{~s}$ the system voltage $U_{s}$ shut down from $1 \mathrm{pu}$ to $0.2 \mathrm{pu}$.

- at time $\mathrm{t}=60$ s the terminal voltage set point $U_{z}$ was changed by step from $1.0 \mathrm{pu}$ to $0.95 \mathrm{pu}$.

\subsection{Two PI+PSS robust decentralized controllers}

(i) PI+PSS - SG excitation control with terminal voltage output feedback: $R_{U}=-(7.3703+8.4564 / s)$,

(ii) - governor control with electrical power output feedback: $R_{T}=-(4.474+6.3072 / s)$

(iii) PSS - excitation controller as the first derivative of load angle: $P S S=0.0190$.

The eigenvalues (eight) of three working points of closed loop system with designed controllers are lying in the following interval EigPI $+P S S=\{-0.8491 \pm$ $0.2989 ; . .-1.5166 \pm 1.2495 \ldots-6.4317 \pm 5.3411 i\}$,

Designed controllers guarantee the closed loop stability for all three working points, minimal value of cost function and all closed loop eigenvalues lying on the left side of complex plane with degree of stability $\alpha=0.6$. Simulation results of above two experiments are given in Figs. 1-6.

\subsection{Two PI gain scheduled+PSS decentralized con- trollers}

(i) PI+PSS gain scheduled controller for excitation control with terminal voltage output feedback. $R_{U}=$ $-\left(6.3492+7.6234 / s+(0.4106+1205 / s) \theta_{1}+(1.3418+\right.$ $\left.\left.0.135 / s) \theta_{2}\right)\right)$

(ii) PI gain scheduled controller for governor control. $R_{T}=\left(-5.3803+6.731 / s+(0.1799+0.148 / s) \theta_{1}+\right.$ $\left.\left.(0.0883+0.1548 / s) \theta_{2}\right)\right)$

(iii) PSS for excitation controller as the first derivative of load angle. $P S S=-0.02370 .0003863 \theta_{1}-0.0008622 \theta_{2}$

The eigenvalues (eight) of closed loop system for designed controllers and frozen gain scheduled variables
(Linear parameter varying system) are lying in the following interval $\theta_{i}=1, i=1,2$, EigGSC $+P S S=$ $\{-0.7963 \pm 0.3029 ; . .-1.7526 \pm 2.8348 \ldots-6.4739 \pm 8.5123 i\}$

Designed guarantee the closed loop stability for all three working points, minimal value of performance and all closed loop eigenvalues lying on the left side of complex plane with degree of stability $\alpha=0.6$. Simulation results of above two experiments are given in Fig.7 to Fig.11.

\section{Comparative studies}

Simulation results of nonlinear turbogenerator model with designed controllers $R_{U}, R_{T}$, and $P S S$ proves that the turbogenerator is stable and all eigenvalues of closed loop system are lying on the left side of vertical line $\alpha=-0.6$ for all working points:

\begin{tabular}{lccccc}
\hline type & Nexp. & Exc.c. & angle & Ter.volt. & act.pow. \\
\hline PI & 1 & 0.131 & 0.192 & 0.0432 & 0.1025 \\
PI & 2 & 33.3 & 33.84 & 35.71 & no overshoot \\
GSC & 1 & 0.1089 & 0.157 & 0.0485 & 0.155 \\
GSC & 2 & 36 & 28 & 26.66 & no overshoot \\
\hline
\end{tabular}

\section{Experiments:}

- at time $t=40 \mathrm{~s}$ two phase short circuits were realized in power system such that within the $t=0.2 \mathrm{~s}$ the system voltage $U_{s}$ shut down from 1 pu to $0.2 \mathrm{pu}$.

- at time $t=60 \mathrm{~s}$ the terminal voltage set point $U_{z}$ was changed by step from $1.0 \mathrm{pu}$ to $0.95 \mathrm{pu}$.

For the first experiment to obtains the coefficient which characterized the damper quality of closed loop system we take the ratio of the third amplitude to the first one of corresponding variable. For the second experiment the value of overshoot (undershoot) in percentage determine the quality of closed loop system. It is well known, that stability of the turbogenerator is determined by the value of load angle and indirectly the value of terminal voltage. Therefore, value of the damper coefficients $\left(D_{x}\right)$ and overshoots $\left(O_{x}\right)$ of load angle and terminal voltage play important role for compare the designed of two controller. In our case their are:

Load angle

$D_{\text {ang }}(P I)=0.192, D_{\text {ang }}(G S C)=0.157, O_{\text {ang }}(P I)=$ $33.84, O_{\text {ang }}(G S C)=28$

Terminal voltage

$D_{\text {volt }}(P I)=0.0432, D_{\text {volt }}(G S C)=0.0485, O_{\text {volt }}(P I)=$ $35.71, O_{\text {volt }}(G S C)=26.66$.

From above results is clear that designed gain scheduled controller is taken over to designed of robust PI controller. While, for both cases the closed loop eigenvalues are lying in the specified LMI region in complex plain what could be shown that both controllers indicate approximately the same quality of dynamic behavior of turbogenerator. But, above table and damper coefficients 


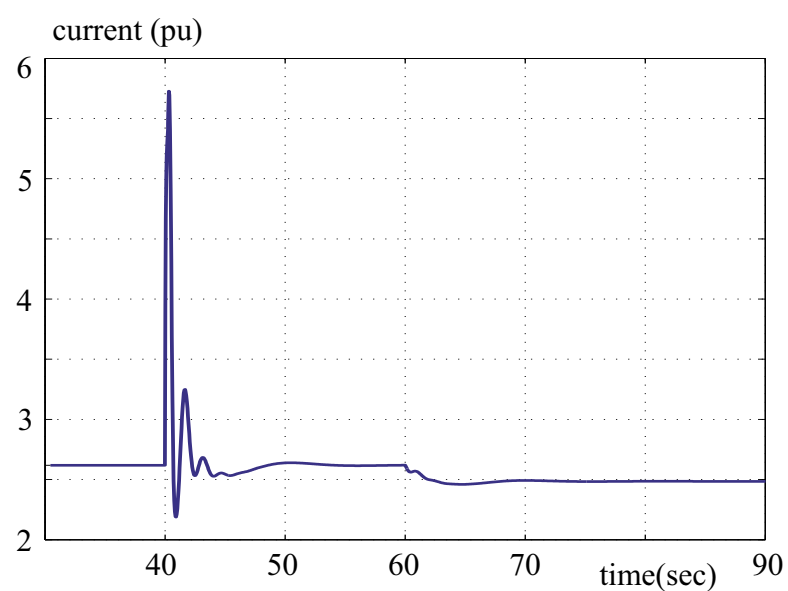

Fig. 7. Excitation current of SG under disturbances-GSC

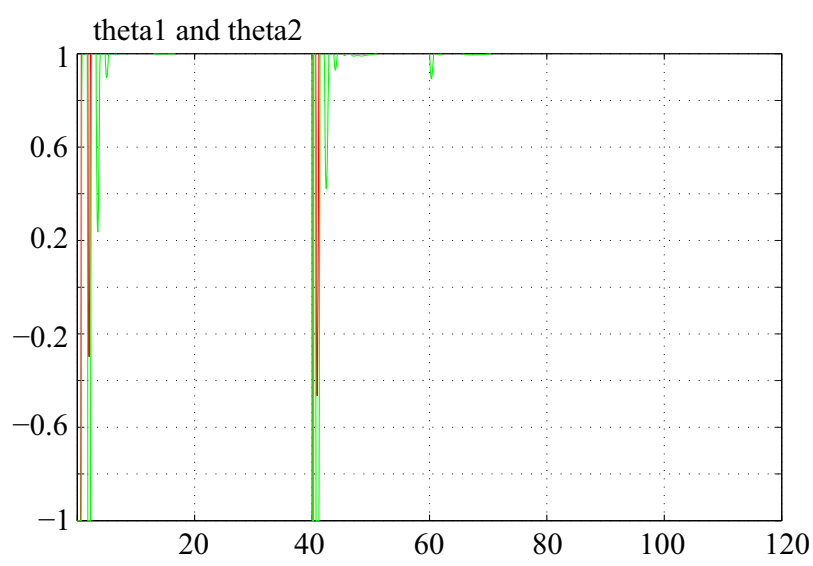

Fig. 9. Excitation current of SG under disturbances-GSC

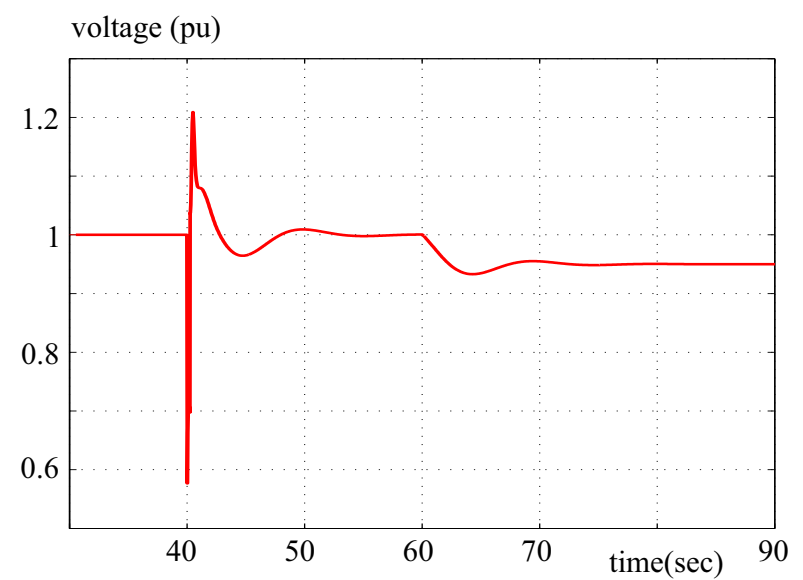

Fig. 11. Load angle and active power of SG dynamic behavior under disturbances-GSC

and overshoots show that gain scheduled controller is better than robust PI controller. If one designed the robust gain scheduled controller the difference of robust GSC and robust PID controllers will be greater in favor to gain scheduled controller.

Calculation results have been obtained using YALMIP with solver penbmi (free).

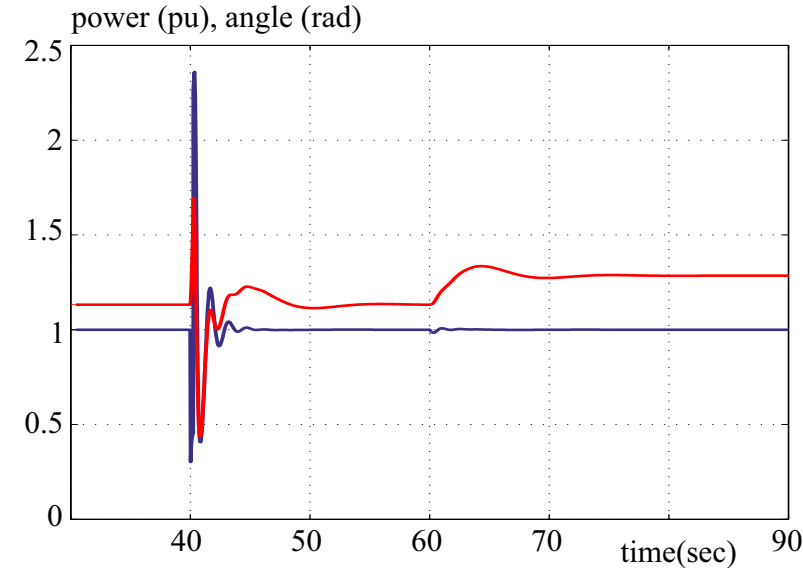

Fig. 8. Load angle and active power of SG dynamic behavior under disturbances-GSC

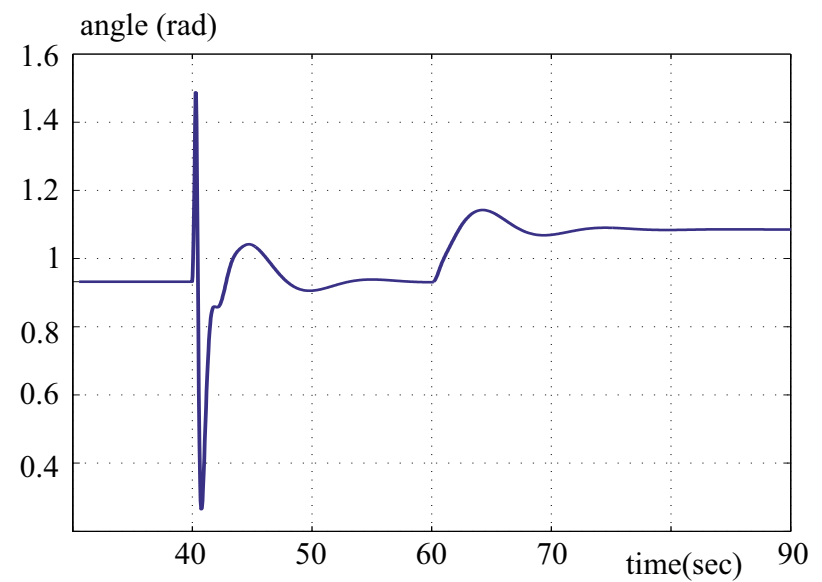

Fig. 10. Load angle and active power of SG dynamic behavior under disturbances-GSC

\section{Conclusion}

In this paper we developed a new design procedure for PI and Gain Scheduled Robust controller with power system stabilizer for turbogenerator using LMI regional regions and/or $\mathrm{H}_{2}$ performance in the form of QSR.

The obtained PI+PSS and GSC+PSS robust controllers with output and state derivative feedback for uncertain polytopic turbogenerator model ensure that all closed -loop eigenvalues lying in the prescribed LMI region and guarantee the minimal value of $H_{2}$ performance. The proposed method guarantee the less conservativeness with respect to privies method due to introducing to the design procedure the new auxiliary matrices. The effectiveness of design procedure is illustrated on the examples to design of mentioned two controllers for turbogenerator. The obtained quality results are summarized in above table which show that damper coefficients and overshoots show of the gain scheduled controller is better than robust PI controller. If one designed the robust gain scheduled controller the difference of robust GSC and robust PID controllers will be greater in favor to gain scheduled controller. 


\section{Acknowledgements}

This work has been supported by the Slovak Scientific Grant Agency, Grant No. 1/0475/16.

\section{REFERENCES}

[1] M. Chilali and P. Gahinet, " $H_{\infty}$ Design with Pole Placement Constraints: An LMI Approach", IEEE Trans on AC vol. 41, no. 3, pp. 358-367, 1996.

[2] D. Peaucelle, D. Alzenier, O. Bachelier, and J. Bernussou, "A New Robust D-Stability Condition for Real Convex Polytopic Uncertainty", Systems Control Letters vol. 40, pp. 21-30, 2000.

[3] M. Chilali, P. Gahinet, and P. Apkarian, "Robust Pole Placement in LMI Regions", IEEE Trans on AC, vol. 44, no. 12, pp. 2257-2270, 1999.

[4] M. Bouhamide and M. A. Danai, "Robust Stabilizer of Electric Power Generator using H1 with Pole Placement Constraints", J. of El. Engineering vol. 56, no. 8, pp. 176-182, 1999.

[5] P. S. Rao and I. Sen, "Robust Pole Placement Stabilizer Design Using Linear Matrix Inequalities", IEEE Trans on Power Systems vol. 15, no. 1, pp. 313-319, 1999.

[6] V. Syrmos Ch. Abdallah, M. Dorato, and K. Grigari-Adis, "Static Output Feedback: A Survey", Conference paper in $\mathrm{Au}$ tomatica vol. 33, no. 2, V1, pp. 837-842, Jan 1995.

[7] P. Gahinet, P. Apkarian, and M. Chilali, "Affine Parameter-Dependent Lyapunov Functions Real Parametric Uncertainty", IEEE Transactions on Automatic Control vol. 41, no. 3, pp. 436-442, Mar 1996.

[8] T. Lahdhiri and A. T. Alouani, "Nonlinear Excitation Control of a Synchronous Generator with Implicit Terminal Voltage Regulation", Electric Power System Research vol. 36, pp. 101-112, 1996.

[9] S. A. Pavlushko, "Automatic Excitation Control of Synchronous Generators as an Effective Means to Ensure the Reliable Parallel Operation of Generation Equipment the United Power System as a Whole", Power Technology Engineering vol. 46, no. 5, pp. 399-406, 1996.

[10] J. Alveraz-Ramirez, I. Cervantes, R. Escarela-Perez, and G. Espriasa-Perez, "A Two-Loop Excitation Control System for Synchronnous Generators", Electrical Power Energy Systems vol. 27, pp. 556-566, 2005.

[11] M. Cao, T. L. Shen, Y. H. Song, and S. W. Mei, "Non-Linear Disturbance Attenuation Controller for Turbo-Generators in Power Systems via Recursive Design", Proc. of the 2002 Int, Conf, on Control Applications University of Groningen, vol. 2, pp. 938-943, 2002.

[12] A. A. Ba-Mugabel and M. A. Abido, "Review of Conventional Power System Stabilizer Design Methods", IEEEGCC Conference IE, Manama, 1-7, March 2006.

[13] E. Ghalipour and S. M. Nosratabadi, "A New Coordination Strategy of SSSC PSS Controllers on Power System using SOA Algorithm based on Pareto Method", Electrical Power Energy Systems, vol. 67, pp. 462-471, 2015.

[14] P. Zhao, W. Yao, J. Wen, L. Jiang, S. Wang, and S. Cheng, "Improved Synergetic Excitation Control for Transient Stability Enhancement Voltage Regulation of Power Systems", Electrical Power Energy Systems vol. 68, pp. 44-51, 2015.

[15] H. Liu, Z. Hu, and Y. Song, "Lyapunov-based Decen-tralized Excitation Control for Global Asymptotic Stability Voltage regulation of Multi-Machine Power Systems", IEEE Trans. on Power Systems vol. 27, no. 4, pp. 2262-2270, 2012.

[16] P. Kundur, M. Klein, G. J. Rogers, and M. S. Zywno, "Application of Power System Stabilizers for Enhancement of Overall
System Stability", IEEE Trans. on Power Systems vol. 4, no. 2, pp. 614-624, 1989.

[17] S. Dechanupaprittha, I. Ngamroo, and Y. Mitani, "Decentralized Design of Robust Power System Stabilizers Considering System Uncertainties", Power Tech IEEE Russia, CD-ROM, 2005.

[18] V. Vesely and A. Kozakova, "Robust PSS Design for a Multivariable Power System", Power Tech Russia, CD-ROM, 2005.

19] H. Liu, J. Qi, J. Wang, and P. Li, "Decentralized Voltage Power Regulation Control of Excitation Governor System with Global Asymptotic Stability", submitted to the IEEE Trans. on Power systems Cite as: arXiv: 1509, 00421[math, OC], 2015.

$20]$ M. Aldeen and F. Grusca, "Multimachine Power System Stabilizer Design based on New LQR Approach", IEE Proc. Gener. Trans. Distrib. vol. 142, no. 5, pp. 494-502, 1995.

[21] V. A. Venikov, "Transient Electromechanical Pro-Cesses in Electric Power Systems", Vyssaja skola Moscow, 1985, (in Russian).

[22] P. Kundur, "Power System Stability Control", McGraw-Hill Inc New York, 1994.

[23] J. Machovski, J. W. Bialek, and J. R. Bumby, "Power System Dynamic Stability Control" John Wiley Sons, Ltd, 2008.

[24] V. Vesely and A. Ilka, "Gain-Scheduled PID Controller Design", Journal of Process Control, vol. 23, pp. 1141-1148, 2013.

25] V. Vesely and D. Rosinova, "Robust PID-PSD Controller Design: BMI Approach", Asian Journal of Control vol. 15, no. 2, pp. 469-478, 2013.

[26] M. R. Esmaili, A. Khodabakhshian, P. Ghaebi Panah, and S. Azirkhan, "A New Robust Multi-Machine Power System Stabilizer Design Using Quantitative Feedback Theory", Procedia Technology vol. 11, pp. 75-85, 2013.

[27] A. E. Leona, J. M. Mauriciob, and J. A. Solsonaa, "MultiMachine Power System Stability Improvement using an Observer-based Nonlinear Controller", Electric Power Systems Research, 89, pp. 204-214, 2012.

28] A. Khodabakhshian and R. Hemmati, "Robust Decentralized Multi-Machine Power System Stabilizer Design using Quantitative Feedback Theory", Electrical Power Energy Systems vol. 41, pp. 112-119, 2012.

[29] V. Vesely, "Robust Decentralized Power System Controller Design", J. of Electrical Engineering vol. 68, no. 5, pp. 349-356, 2017.

[30] Y. Tamura ansd and N. Yorino, "Possibility of Auto-HeteroParametric Resonances in Power Systems their Relationship with Long-Term Dynamics", IEEE Trans on Power Systems vol. PWRS-2, no. 4, pp. 890-896, Nov 1987.

[31] D. J. Leith and W. E. Leithead, "Survey of Gain-Scheduling Analysis Design", International Journal of Control vol. 73, no. 11, pp. 1001-1025.

[32] M. Sato and D. Peaucelle, "Gain-Scheduled Output-Feedback Controllers using Inexact Scheduling Parameters for Continuous-Time LPV Systems", Automatica vol. 49, no. 4, pp. 1019-1025,April 2013.

[33] C. W. Scherer, "Gain Scheduling Control with Dynamic Multipliers by Convex Optimization", Siam Journal on Control Optimization, vol. 53, no. 3, pp. 1224-1249, 2015.

34] V. Vesely and A. Ilka, "Design of Robust Gain-Scheduled PI Controllers", Journal of Franklin Institute, vol. 352, pp. 1476-1494, 2015.

[35] W. J. Rugh and J. S. Shamma, "Survey Research on Gain Scheduling", Automatica vol. 36, no. 10, pp. 1401-1425, Oct 2000.

Received 4 December 2018 